\title{
Observational constraints on the afterglow of GRB 020531
}

\author{
A. Klotz ${ }^{1}$, M. Boër ${ }^{1}$, and J. L. Atteia ${ }^{2}$ \\ ${ }^{1}$ Centre d'Étude Spatiale des Rayonnements, Observatoire Midi-Pyrénées (CNRS-UPS), BP 4346, \\ 31028 Toulouse Cedex 04, France \\ 2 Laboratoire d'Astrophysique, Observatoire Midi-Pyrénées (CNRS-UPS), 14 avenue E. Belin, \\ 31400 Toulouse, France
}

Received 20 November 2002 / Accepted 17 January 2003

\begin{abstract}
We present the data acquired by the TAROT automated observatory on the afterglow of GRB 020531. Up to now, no convincing afterglow emission has been reported for this short/hard GRB at any wavelength, including X-ray and optical. The combination of our early limits, with other published data allows us to put severe constraints on the afterglow magnitude and light curve. The limiting magnitude is 18.5 in $R$ band, $88 \mathrm{~min}$ after the GRB, and the decay slope power law index could be larger than 2.2.
\end{abstract}

Key words. gamma-ray : bursts

\section{Introduction}

Since their first detection by van Paradijs et al. (1997), gammaray burst (GRB) optical afterglows have been detected in about $40 \%$ of the sources displaying an X-ray afterglow. The fireball model (Rees \& Mészáros 1992; Mészáros \& Rees 1997; Panaitescu et al. 1998) has been established as a standard tool to interpret these observations. In this framework the afterglow emission is described as synchrotron and inverse Compton emission of high energy electrons accelerated during the shock of an ultra-relativistic shell with the external medium, while the prompt emission is due to the internal shocks produced by shells of different Lorentz factors within the relativistic blast wave (see Piran 1999 for a review). Both the prompt radiation and early afterglow phases provide critical information to establish the physical processes at work during the burst itself, as well as the physical conditions of the surrounding environment (Kumar \& Panaitescu 2000; Kumar \& Piran 2000). There is a general consensus that the fireball plasma is constituted by $\mathrm{e}^{-} \mathrm{e}^{+}$ pairs and $\gamma$-ray photons, however the ultimate energy reservoir and the detailed radiation mechanisms are still a challenge to theoretical models.

The situation of $60 \%$ of the GRB afterglows which are not observed at optical wavelengths (called dark GRBs) is not clear. As it has been shown in Boër \& Gendre (2000), the optical flux is not correlated with the intensity of the $\mathrm{X}$-ray afterglow, nor with the distance. Generally speaking the absence of an optical transient associated with a GRB can be attributed to four, non exclusive, reasons, namely 1) the distance of the source, though this is obviously not the general

Send offprint requests to: A. Klotz, e-mail: klotz@cesr.fr case, 2) the absorption of the visible light by a dense medium (i.e. dust), 3) the rapid decay of the optical afterglow, and 4) the intrinsic faintness of the source at long wavelengths (i.e. optical, NIR...). However, a few reports of near IR and optical non-detection of GRB afterglows show that hypothesis 2 is not the main reason (see e.g. GRB 010214, Piro 2001 and subsequent GCN circular available at the URL http://gcn.gsfc.nasa.gov/gcn/other/010214.gcn3). In the absence of rapid simultaneous X-ray and optical measurements, hypotheses 3 and 4 are difficult to evaluate.

It should be noted that for the sub-class of GRBs that exhibit a short duration and a hard spectrum, usually called short/hard GRB (Dezalay et al. 1996; Kouveliotou et al. 1993), no optical counterpart has been detected yet (Hurley et al. 2002a; Gorosabel et al. 2002) excepted in the case of GRB 000313 (Castro-Tirado et al. 2002a published $R=9.4$ at 4 min after GRB). However the reality of the afterglow candidate for GRB 000313 is questionable because it is seen in only one image. The usual no optical counterpart detection is largely due to the scarcity of the observations. If this appears a "general" law, it can be the indication of a different geometry (as viewed from the observer) or of another mechanism for the emission of the afterglow (e.g. Shanthi et al. 1999). Hence, it is important to get rapid and deep measures (or upper limits) on the afterglow emission for GRB sources of all classes, and in particular for the short GRBs.

In this Paper we report on the early observations of GRB 020531 performed with the automatic TAROT observatory (Bringer et al. 1999). Our data, combined with the data from other telescopes strongly constrain both the magnitude and the decay slope index of the optical counterpart, if any. 


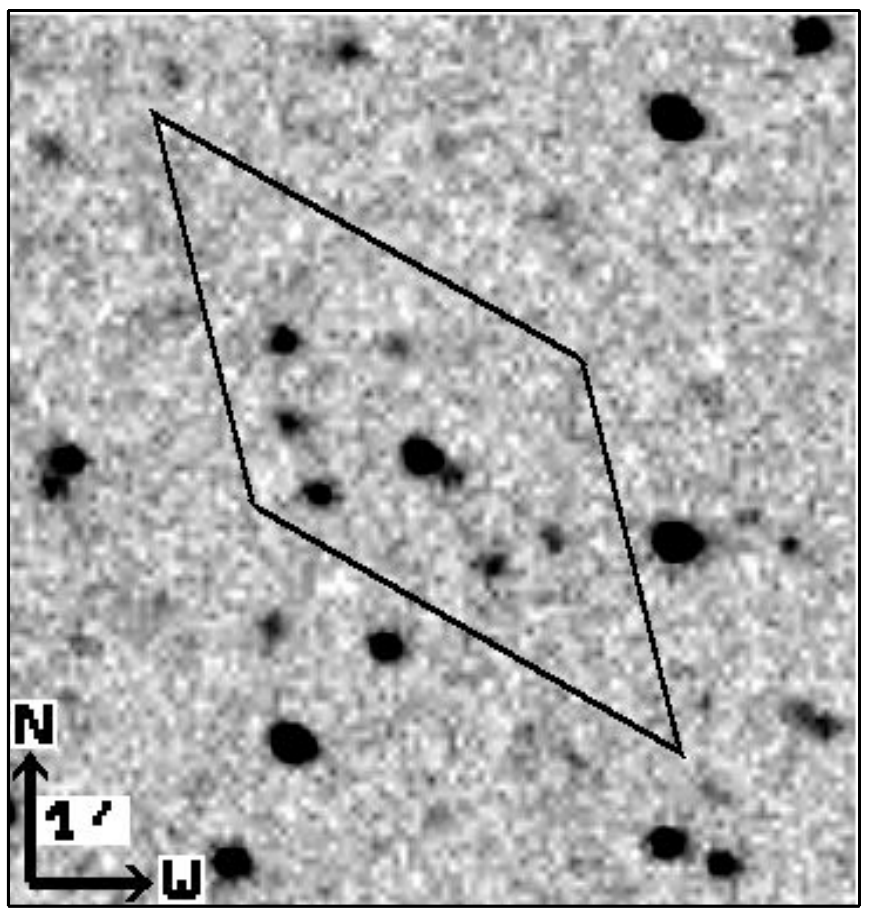

Fig. 1. A sub-image of the TAROT composite image (11 frames of duration $30 \mathrm{~s}$ each). The parallelogram is the last IPN error box (from GCNC 1461, Hurley et al. 2002b). The limiting magnitude is 18.5 ( $R$ band).

\section{Observations}

\subsection{Detection and follow up of the burst}

The High Energy Transient Explorer satellite (HETE, Ricker et al. 2000) detected GRB 020531 with the FREGATE and WXM instruments on May 31, 2002 at $0 \mathrm{~h} 26 \mathrm{~min} 18.73$ UTC (Ricker et al. 2002). This event is a short/hard GRB: $t_{90}=$ $0.94 \mathrm{~s}, t_{50}=0.45 \mathrm{~s}$, and fluence is $8 \times 10^{-7} \mathrm{erg} \mathrm{cm}^{-2}$ in the FREGATE 50-300 keV band. The absolute localization was not performed by the flight software and the preliminary coordinates were computed by a ground analysis. The GRB Coordinates Network (GCN - Barthelmy 1997) broadcasted the position at $1 \mathrm{~h} 54 \mathrm{~min} 22 \mathrm{~s}$ UT. Additional information about the GRB localization can be found in Lamb et al. 2002. Twenty-five GCN circulars (GCNC) were published on this event between May 31 and July 25, 2002. In the first very early reports, it appears that no unambiguous optical counterpart was recorded. The final error box given by the Inter Planetary Network (IPN) published on the July 10th 2002 (Hurley et al. 2002b). In this area, four faint sources were detected by the Chandra satellite ACIS-I array (Butler et al. 2002) five days after GRB. The connection of one of these X-ray sources with the gamma-ray transient remains to be confirmed.

Up to now, none of the suggested optical counterparts of GRB 020531 has been confirmed. In this study we present the data acquired with the TAROT observatory. Our limits are compared with the limiting magnitudes obtained by other observers at different times after the GRB. Given that our data were obtained only $88 \mathrm{~min}$ after the burst itself, we can infer strong limits both on the optical counterpart magnitude and decay slope.

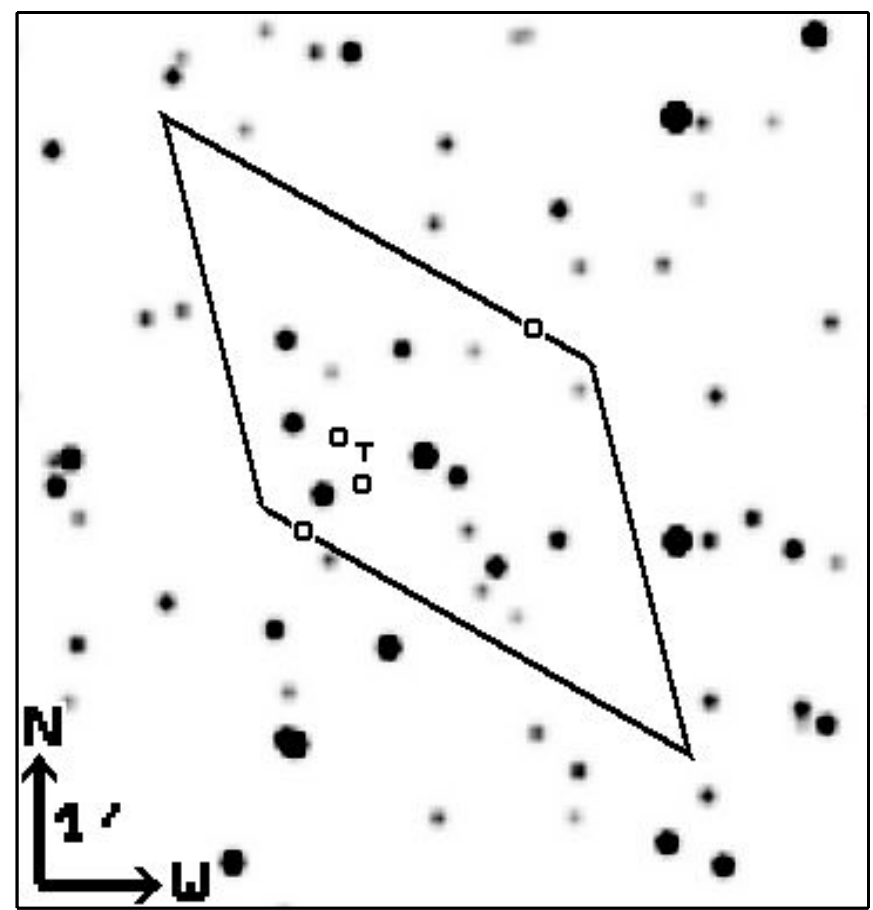

Fig. 2. A synthetic Cousins $R$ image of the same field as the TAROT image in Fig. 1. Magnitude values are taken from the $B V R_{\mathrm{c}} I_{\mathrm{c}}$ allsky photometry, posted by Henden 2002 in GCNC 1422 . Only stars brighter than $R_{\mathrm{c}}=18.5$ are displayed, i.e. up to the limiting magnitude of the TAROT image. Circles indicate the positions of the Chandra observatory X-ray sources (from GCNC 1415, Butler et al. 2002). The T symbol is the location of the Tarot-C source (from GCNC 1420, Klotz et al. 2002).

\subsection{Tarot observations}

TAROT is a fully autonomous $25 \mathrm{~cm}$ aperture telescope installed at the Calern observatory (Observatoire de la Côte d'Azur - France). Its $2^{\circ}$ field of view ensures the total coverage of HETE error boxes. This telescope is devoted to very early observations of GRB optical counterparts. A technical description of TAROT can be read in Bringer et al. (1999) and in Bringer et al. (2001). The CCD camera is based on a THX7899 Thomson chip. It is placed at the newtonian focus. The focal length is $0.81 \mathrm{~m}$ and the pixel size is 14 microns. The spatial sampling is $3.5 \mathrm{arcsec} / \mathrm{pixel}$. The readout noise is 13 electrons rms and the actual gain is 3.6 photo-electrons/adu. The main feature of this camera is its very short readout time: $2 \mathrm{~s}$ to read the entire $2048 \times 2048$ matrix with no binning.

The first image was taken by TAROT less than $6 \mathrm{~s}$ after the position of GRB 020531 was provided by the GCN. A series of 11 unfiltered images of $30 \mathrm{~s}$ was then taken. An automatic preprocessing software gave scientific images in the following minutes. We compared them to the Digital Sky Survey (DSS) images. We concluded quickly that no bright new source was present. The limiting magnitude of the individual images, in the Cousins $R$ band, is about 16.7.

Then we coadded the 11 images to improve the signal to noise ratio (see Fig. 1). A limiting magnitude of 18.5 (compared to the $R$ Cousins band) is reached. This limiting magnitude is estimated from comparison with a set of synthetic 
Table 1. Log of the published values of the limiting magnitudes, presented in the chronological order. The first column is the date from GRB (in days). The second is the limiting $R$ magnitude of the image. The third is the GCN circular index of the publication.

\begin{tabular}{cccc}
\hline \hline Date & $R$ lim & GCNC & Instrument \\
\hline 0.0000 & 8 & 1430 & BOOTES-1 $(D=0.06 \mathrm{~m})$ \\
0.0208 & 8 & 1430 & BOOTES-1 $(D=0.06 \mathrm{~m})$ \\
0.0654 & 18.5 & 1408 & TAROT $(D=0.25 \mathrm{~m})$ \\
0.0997 & 17.7 & 1406 & D. West $(D=0.20 \mathrm{~m})$ \\
0.1512 & 17.5 & 1404 & Super-LOTIS $(D=0.60 \mathrm{~m})$ \\
0.1831 & 18 & 1400 & NEAT $(D=1.2 \mathrm{~m})$ \\
0.1859 & 18 & 1401 & SDSS $(D=0.5 \mathrm{~m})$ \\
0.1873 & 20.5 & 1405 & KAIT $(D=0.8 \mathrm{~m})$ \\
0.3790 & 18 & 1401 & SDSS $(D=0.5 \mathrm{~m})$ \\
0.9017 & 24.7 & 1433 & INT $(D=2.5 \mathrm{~m})$ \\
1.1417 & 23.6 & 1434 & Baade $(D=6.5 \mathrm{~m})$ \\
1.2352 & 20.5 & 1405 & KAIT $(D=0.8 \mathrm{~m})$ \\
2.9717 & 25.2 & 1433 & INT $(D=2.5 \mathrm{~m})$ \\
5.4317 & 25.5 & 1434 & Subaru $(D=8.2 \mathrm{~m})$ \\
10.1117 & 24.0 & 1434 & Baade $(D=6.5 \mathrm{~m})$ \\
\hline
\end{tabular}

images computed from the $B V R_{\mathrm{c}} I_{\mathrm{c}}$ USNOFS all-sky photometry of field (Henden 2002). In Fig. 2, only stars brighter than $R_{\mathrm{c}}=18.5$ are plotted.

Three TAROT sources, afterglow candidates, were published in the GCN circulars: sources A and B (Boër et al. 2002) and C (Klotz et al. 2002).

Source A, RA $=15 \mathrm{~h} 14 \mathrm{~min} 51 \mathrm{~s}$ Dec $=-19^{\circ} 25^{\prime} 06^{\prime \prime}$ (J2000.0), $R=17.4$, cannot be the asteroid number 2 mentioned by Li et al. (2002) in the GCNC 1405, as it was supposed by Boër et al. (2002) in the GCNC 1408. The reason is that it lies in the opposite side of the apparent motion published by Li et al. (2002). Source B, RA $=15 \mathrm{~h} 14 \mathrm{~min} 57 \mathrm{~s}$ Dec $=-19^{\circ} 28^{\prime} 12^{\prime \prime}(\mathrm{J} 2000.0), R=17.1$, is a known star visible in DSS and various other images. Anyway, A and B sources lie outside the IPN error box.

Source C, RA $=15 \mathrm{~h} 15 \min 12 \mathrm{~s}$ Dec $=-19^{\circ} 24^{\prime} 24^{\prime \prime}$ (J2000.0), $R \geq 18.5$, is considered as the best TAROT image candidate in the IPN error box. We reprocessed the raw images using the calibration frames taken both before and after the night of May 30-31, 2002, and we obtained a fainter source at the position of the source $\mathrm{C}$ on the new refined co-added images. This meant that source $\mathrm{C}$ could be a group of "hot pixel" badly corrected by the automatic preprocessing which uses only the calibration frame taken during the preceding day, to produce synthetic calibration data.

Other fuzzy patches are also seen in the image of TAROT presented in Fig. 1. All of these patches can be related to known stars fainter than $R_{\mathrm{c}}=18.5$. However, as the TAROT image is unfiltered, it is not surprising to find these stars (color effects).

\subsection{Other observations}

The data reported in various GCN circulars are summarized in Table 1. The first column is the delay, in fraction of day,

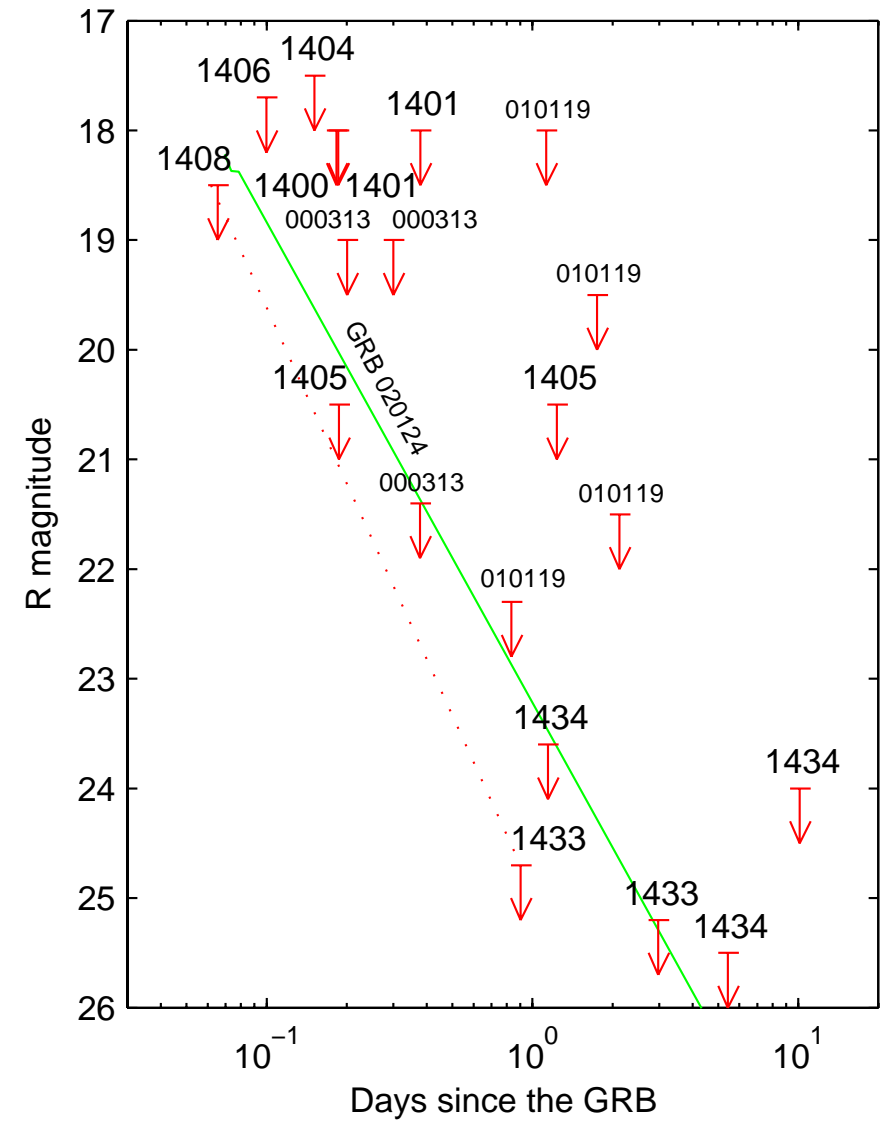

Fig. 3. Reported upper limits of the magnitude (from Table 1) of the optical afterglow of GRB 020531 (arrows, with the GCN circular number). Limiting magnitudes of the two other early observations from GCN $1430(R>8)$ lie far outside upper the panel. For comparison, we added some data, labeled 010119 and 000313 , respectively for the upper limits of the short/hard GRB 010119 (Gorosabel et al. 2002) and GRB 000313 (Castro-Tirado et al. 2002a). We plotted, as a solid line, the light curve of the dim afterglow of the long burst GRB 020124 (Berger et al. 2002). The dotted line represents the upper limit for the brightness of the afterglow, assuming a constant decay slope.

between the burst and the beginning of the observation, the second column gives the limiting magnitude, the third column indicates the GCN circular in which the data was reported, and the last one the instrument used as well as its aperture. For early observations ( $<1$ day after GRB), only small aperture telescopes (i.e. $<2$ meters) scanned the field. During this delay, the better limiting magnitude is 20.5 from the Katzman Automatic Imaging Telescope (KAIT, Li et al. 2002). From later observations ( $>1$ day), the most constraining limiting magnitude is 24.7 at 0.9017 day, obtained by the Isaac Newton Telescope at La Palma (Salamanca et al. 2002). The limiting magnitudes, summarized in Table 1, are displayed in Fig. 3.

\section{Discussion}

Up to now, no afterglow of a short/hard GRB was detected. However, it is possible to get some constraints on the optical light curve. The best limits to constrain the light curve for the afterglow of GRB 020531 comes from TAROT, KAIT, and INT data. If GRB 020531 was followed by an optical afterglow, 
its light curve must lie in the left part of Fig. 3, below the dashed line.

Before GRB 020531, the earliest optical observations of a short/hard GRB were obtained on GRB 000313 (Castro-Tirado et al. 2002a) and on GRB 010119 (Gorosabel et al. 2002).

The decay slope index $\alpha$ for an afterglow of a short/hard GRB (assuming flux proportional to $t^{-\alpha}$ ) is now more constrained by GRB 020531 observations. Typical long GRBs afterglow decays are between 0.7 and 1.8, marginally higher than 2 (i.e. GRB 980519, Vrba et al. 2000). Concerning GRB 020531, if the flux of the afterglow was about the limiting magnitude of TAROT $(R=18.5$ at 1.47 hour after the burst), then its decay slope $\alpha$ must be $>2.2$. This slope value is in accordance with the GRB 000313 results (Castro-Tirado et al. 2002a). If the afterglow of GRB 020531 was fainter at 1.47 hour after the burst, the decay slope should have a lower value. If the afterglow of GRB 020531 was $R=9.4$ at 4 min after GRB (the same as the detection of GRB 000331), the TAROT observation constrains the slope to be higher than 2.6.

Comparing to the dimmest long GRBs, e.g. GRB 020124 (Berger et al. 2002, see Fig. 3), it implies that the afterglow of GRB 020531 must be fainter. The TAROT upper limit measurement constrains the afterglow to be very dim. This result is correlated to the $50-300 \mathrm{keV}$ fluence which is one decade fainter than those of typical long GRBs.

If the afterglow exists and decays with a $t^{-\alpha}$ law, and if the source flux was about the limiting magnitude of late observations, one can calculate $R=22.0$ at 1.47 hour after the GRB (TAROT observations) assuming $\alpha=1.2$ (the typical case). Obviously, the afterglow can be even fainter if it is dimer than the limiting magnitude of late observations. As a consequence, plans for future searches of afterglows of short/hard GRBs can be adressed: large aperture telescopes, equiped by wide field cameras, should observe early stages (until 1 hour after GRB). Small aperture telescopes could also contribute if they shoot until 15 min after GRB with a limiting magnitude $R>18$.

\section{Conclusion}

The afterglow of GRB 020531, if it exists, is very dim, compared to the observed optical counterparts of long GRBs. If the optical counterpart of GRB 020531 is typical of short/hard GRBs, it means that these kinds of GRBs are associated to very dim afterglows or no afterglow at all. The observations suggest that the decay slope $\alpha$ could be larger than 2 .

It must be mentioned that dim afterglows can be localized only by early optical observations (case of GRB 020124 afterglow, found at 1.67 hour after the GRB).

Of course the possibility that GRB 020531 had no afterglow cannot be excluded. This proves the need to get more sensitive observations of the afterglow, as early as possible after the main event. The TAROT observatory demonstrated that this is possible, provided that the alert is sent quickly by the instrument. The increase in the HETE performances, the recent successful launch of the Curie-INTEGRAL satellite, as well as the perspective of the SWIFT GRB dedicated satellite gives hope that rapid observations of GRB optical counterparts will be soon possible, as it was the case with BATSE (Akerlof et al. 1999; Boër et al. 2001; Castro-Tirado et al. 1999; Park et al. 1999).

Acknowledgements. The Télescope à Action Rapide pour les Objets Transitoires (TAROT) has been funded by the Centre National de la Recherche Scientifique (CNRS), Institut National des Sciences de l'Univers (INSU) and the Carlsberg Fundation. It has been built with the support of the Division Technique of INSU (INSU/DT). The TAROT CCD camera was built by a collaboration between the CESR and the CEMES. We thank the technical staff associated with the TAROT project: G. Bucholtz, J. Esseric, A. Mayet, A. M. Moly, F. Morand, M. Nexon, H. Pinna, and C. Pollas. We thank Holger Pedersen and the referee for their remarks.

\section{References}

Akerlof, C., Balsano, R., Barthelemy, S., et al. 1999, Nature, 398, 400 Barthelmy, S. 1997, Proceedings of the 4th Huntsville Symp., ed. C. A. Meegan, R. D. Preece, \& T. M. Koshut, AIP Conf. Proc., 428, 99

Berger, E., Kulkarmi, S. R., Bloom, J. S., et al. 2002, ApJ, 581, 981

Boër, M., \& Gendre, B. 2000, A\&A, 361, L21

Boër, M., Atteia, J. L., Bringer, M., et al. 2001, A\&A, 378, 76

Boër, M., Klotz, A., Atteia, J. L., et al. 2002, GCNC 1408

Bringer, M., Boër, M., Peignot, C., Fontan, G., \& Merce, C. 1999, A\&AS, 138, 581

Bringer, M., Boër, M., Peignot, C., Fontan, G., \& Merce, C. 2001, Exper. Astrophys., 12, 34

Butler, N., Dullighan, A., Ford, P., et al. 2002, GCNC 1415

Castro-Tirado, A. J., Soldán, J., Bernas, M., et al. 1999, A\&AS, 138, 583

Castro-Tirado, A. J., Castro Cerón, J. M., Gorosabel, J., et al. 2002a, A\&A, 393, L55

Castro-Tirado, A. J., Castro Cerón, J. M., de Ugarte Postigo, A., et al. 2002b, GCNC 1430

Dezalay, J. P., Lestrade, J. P., Barat, C., et al. 1996, ApJ, 471, L27

Gorosabel, J., Andersen, M. I., Hjorth, J., et al. 2002, A\&A, 383, 112

Henden, A. 2002, GCNC 1422

Hurley, K., Berger, E., Castro-Tirado, A., et al. 2002a, ApJ, 567, 447

Hurley, K., Cline, T., Mitrofanov, I., et al. 2002b, GCNC 1461

Klotz, A., Boër, M., \& Atteia, J. L. 2002, GCNC 1420

Kouveliotou, C., Meegan, Ch. A., Fishman, G. J., et al. 1993, ApJ, 413, L101

Kumar, P., \& Panaitescu, A. 2000, ApJ, 541, L9

Kumar, P., \& Piran, T. 2000, ApJ, 535, 152

Lamb, D. Q., et al. 2002, in preparation

Li, W. D., Filippenko, A. V., \& Chornock, R. 2002, GCNC 1405

Mészáros, P., \& Rees, M. 1997, ApJ, 476, 232

Panaitescu, A., Mészáros, P., \& Rees, M. 1998, ApJ, 503, 314

Park, H. S., Porratta, R. A., Williams, G. G., et al. 1999, A\&AS, 138, 577

Piran, T. 1999, Phys. Rep., 314, 575

Piro, L. 2001, GCNC 932

Rees, M., \& Mészáros, P. 1992, MNRAS, 258, 41

Ricker, G. R., \& HETE Science Team 2000, Am. Astron. Soc. Meet., 197, 2501

Ricker, G. R., Atteia, J.-L., Kawai, N., et al. 2002, GCNC 1399

Salamanca, I., Rol, E., Tanvir, N., \& Kaper, L. 2002, GCNC 1433

Shanthi, K., Rao, A. R., Bhat, C. L., \& Vahia, M. N. 1999, Bull. Astr. Soc. India, 27, 195

van Paradijs, J., Groot, P. J., Galama, T., et al. 1997, Nature, 386, 686

Vrba, F. J., Henden, A. A., Canzian, B., et al. 2000, ApJ, 528, 254 